\title{
El Desarrollo Sostenible de la Comunidad ${ }^{1}$
}

\author{
M.E. Swisher, Sandra Rezola and James Sterns ${ }^{2}$
}

\section{Resumen}

Este documento se trata del concepto del desarrollo sostenible de la comunidad y como la implementación de proyectos enfocados en el desarrollo sostenible pueden mejorar la calidad de la vida en una comunidad. Se examinan los conceptos básicos del desarrollo sostenible y se proveen ejemplos de las amenazas a la calidad de la vida que el desarrollo tradicional puede causar. El documento señala seis pasos que se puede usar para lograr el desarrollo sostenible.

\section{El Desarrollo Sostenible de la Comunidad}

\section{Una Comunidad Sostenible:}

Proteja el Ambiente: La toma de decisiones se enfoca en la reducción de los impactos del crecimiento de la población y del desarrollo en los recursos naturales y el ambiente.

Es Económicamente Productivo: Los miembros de la comunidad invierten su capital local para sostener los recursos humanos y naturales locales y para lograr un retorno en sus inversiones.

Promueve la Justicia Social: El acceso equitable a los recursos y al proceso de la toma de decisiones estimula la distribución de bienes por todos los sectores de la comunidad.

Imagínese una comunidad donde el agua y el aire están limpios, donde hay agua suficiente para todos, donde todo el mundo puede conseguir alimentos saludables y sanos producidos localmente, donde la vida silvestre abunda y donde el paisaje es agradable. En esta comunidad, todos los ciudadanos participan activamente en la toma de decisiones y hay un espíritu de colaboración. Los habitantes afectan directamente el futuro de la comunidad. Se renueve el centro comercial del pueblo, se reduce el alargamiento espacial de la ciudad y los ciudadanos son orgullosos de identificarse como miembros de la comunidad. Un sistema eficiente del transporte reduce la congestión y la contaminación producida por los carros. Se economiza el costo del transporte y es fácil viajar a las empresas y oficinas. Los miembros de la comunidad ganan un sueldo justo. Un fuerte énfasis en la educación y el entrenamiento promueve una alta calidad de vida actualmente y asegura oportunidades abundantes para la juventud.

Todo lo anterior no es solamente imaginario. Al contrario, comunidades por todo el país están creando visiones parecidas del desarrollo sostenible para sus comunidades. Sarasota, Florida, ofrece

1. This document is FCS7213-Span, one of a series of the Department of Family, Youth and Community Sciences, Florida Cooperative Extension Service, IFAS, University of Florida, Gainesville FL 32611: First published: September 2003. English version reviewed by Jerry Culen, Ph.D., associate professor and Amy Simmone, Ph.D., assistant professor, Department of Family, Youth and Community Sciences, and Burl Long, Ph.D., professor, Department of Food and Resource Economics, University of Florida, Gainesville, Florida, 32611. Please visit the EDIS Web site at http://edis.ifas.ufl.edu

2. M.E. Swisher, Ph.D., associate professor, Department of Family, Youth and Community Sciences, Sandra Rezola, M.S., Institute of Food and Agricultural Sciences, and James Sterns, Ph.D., assistant professor, Department of Food and Resource Economics, University of Florida, Gainesville FL 32611. 
un ejemplo. Esta comunidad inició el proceso de crear una visión del desarrollo sostenible para Sarasota en los años 1990 (20/20 Foresight Sustainable Development Visioning Process). El proceso fue la respuesta de la comunidad a muchos problemas, entre ellos el crecimiento de la población y del área urbana, la congestión y la escasez de agua. Se formaron colaboraciones entre organismos cívicos, públicos y privados para establecer metas para el desarrollo sostenible. Desde 1990, se han llevado a cabo tres conferences con el fin de guiar el desarrollo en el centro de Sarasota hasta el año 2040. Una colaboración entre el Servicio de Extensión del Cantón de Sarasota y la Fundación para la Casa Floridana formó el Centro Educativo de la Casa Floridana para promover el desarrollo sostenible en las urbanizaciones en el cantón. Para reducir el uso de plaguicidas y la exposición de los miembros de la comunidad a ellas, el cantón estableció un programa de manejo integrado de plagas para todos las edificaciones y tierras que pertenecen al cantón en 1991. También, el cantón estableció el programa de reciclaje de basura más comprehensiva del estado y se dedicó a la restauración de la calidad de la Bahía de Sarasota, con apoyo de Mott Marine Laboratory. Otras actividades incluyeron el establecimiento de centros, fundaciones, programas educativas, y programas para la adquisición de tierra, el desarrollo económico, la conservación de recursos y la protección del ambiente.

Otras comunidades por toda la nación también se están implementando sus visiones del desarrollo sostenible. El desarrollo sostenible de la comunidad es relevante para la Florida en la actualidad, no en un futuro lejos.

\section{Tendencias que Amenazan las Comunidades de Florida}

La población del estado de Florida se ha expandido enormemente, de aproximadamente 2.8 millones en 1950 a 15.6 millones en 2000. El Censo de los Estados Unidos indica que la inmigración, la alta tasa de nacimiento y la migración nacional puede aumentar la población de Florida a 20.7 millones en el año 2025. Entre 1970 y 1990, los áreas urbanas de Florida se expandieron para ocupar más de un millón de acres de tierras rurales, incluyendo hábitats naturales, tierras agrícolas y espacios abiertos, reemplazandolos en centros comerciales y urbanizaciones (Kolankiewicz y Beck, 2000). El crecimiento sin control ha causado problemas ambientales, sociales y económicos que amenazan las mismas calidades del estado que motivaron los inmigrantes a venir a Florida y que tanto el nuevo habitante como el viejo valúa tanto.

Los recursos acuáticos provienen un buen ejemplo. La Florida tiene la tasa más alta de uso doméstico de agua en el país, la tasa segunda más alta para la minería, y la cuarta más alta para uso público. Debido principalmente al crecimiento de la población, se cree que no habrá agua suficiente para llenar las necesidades de la población por el año 2020. Se imponen restricciones en el uso de agua, un resultado de la competición entre la comunidades del estado y entre los habitantes de las ciudades y los agricultores. Además, la escorrentía en los pueblos y de las tierras agrícolas, la contaminación industrial y municipal y la construcción contribuyen a los problemas de contaminación del agua por todo el estado.

\section{El Enfoque en la Comunidad}

La Florida, como lo demás de la nación, requiere de un plan de acción para mejor llevar a cabo el desarrollo social, ambiental y económico. Visto al nivel de todo el estado, los problemas puedan 
parecer tantos y tan complejos que no se puede tomar acción efectiva. Sin embargo, los problemas como el crecimiento urbano, la degradación ambiental y la injusticia social se vuelvan muy reales y personales en las comunidades donde vivimos y trabajamos. Se enfrentan estos problemas al nivel de la comunidad y se los pueden resolver al nivel local. Por ejemplo, la Comisión de las Naciones Unidas para el Ambiente y el Desarrollo define el desarrollo sostenible como el desarrollo que "llena las necesidades del presente sin comprometer la habilidad de las generaciones futuras de llenar sus propias necesidades." Al nivel de la comunidad, estas metas abstractas se transforman en la acción concreta y efectiva.

Cada comunidad tiene que desarrollar su propia visión y plan de acción. No hay una sola definición del desarrollo sostenible de la comunidad porque cada comunidad tiene sus propias características y retos. Sin embargo, las comunidades sostenibles comparten ciertas aspectos y características: la seguridad económicas, la protección del ambiente, la justicia social y una dedicación al bienestar de las generaciones futuras.

Las comunidades sostenibles reconocen los enlaces entre la economía, la estructura social y la calidad del ambiente. Entienden que los programas y las políticas que estimulan un sólo aspecto del desarrollo, sea el crecimiento económico, el progreso social o la protección del ambiente, sin tomar en cuenta los otros componentes, no promueven el progreso sostenido para la comunidad. Por lo tanto, se toman en cuenta estos tres componentes juntos en el proceso de la toma de decisiones. Por ejemplo, los consumidores toman en cuenta el papel de los agricultores local en proteger el ambiente y su contribución a la economía local cuando deciden donde comprar los alimentos. Los profesionales dedicados al manejo de los recursos naturales consideran no solamente la capacidad de los recursos naturales para suportar la comunidad, sino también la capacidad social y la infraestructura requerida para mantenerla. Los líderes de la comunidad piensan no solamente en el corto plazo y en sus desacuerdos, sino en el largo plazo y en sus intereses en común.

\section{La Acción}

Para formular una estrategia para el desarrollo sostenible, los miembros de la comunidad tiene que creer que ellos mismos pueden resolver sus problemas y crear su propio futuro. La red de una araña parece frágil. Sin embargo, es capaz de sostener mucha fuerza sin dañarse. El proceso de fortalecer la capacidad de la comunidad es parecido al proceso de tejar una red, una red que crea enlaces sociales por toda la comunidad, que provee apoyo para todos sus miembros y que refuerza la cooperación y la colaboración entre las instituciones, las organizaciones y los negocios presentes en la comunidad. Aunque pueda ser pequeña al principio, la red se extiende y se incorporan más y más miembros de la comunidad. Se aumenta la cohesión en la comunidad y su resiliencia por las alianzas, la colaboración y una visión común del futuro.

Las iniciativas para el desarrollo sostenible, como aquella de Sarasota, tratan de los asuntos importantes al nivel local. Cada comunidad tiene que formular su propia estrategia para el desarrollo sostenible. Sin embargo, los siguientes pasos puede ayudar a su comunidad en el desarrollo y la implementación de una visión común del futuro.

1. Crear Consenso.

2. Evaluar los Recursos Disponibles. 
3. Desarrollar Una Visión Común.

4. Identificar Indicadores del Progreso.

5. Identificar Fuentes de Apoyo.

6. Implementación, Evaluación y Ajuste.

\section{Literatura Citada}

Kolankiewicz, L. y Beck, R. 2000. Sprawl in Florida. Sprawl City. Retrieved from http://www.sprawlcity.org

Mishel, L.; Bernstein, J. y Schmitt, J. 2000. The State of Working America 2000-01. Cornell University Press, Ithaca, NY. 153 pág.

Censo de los Estados Unidos. 2000. Profile of Selected Economic Characteristics. U.S. Census Bureau, Washington, D.C. 